\title{
Détection luminale des micelles lipidiques
}

Olivier BEASLAS

Carine CUEILLE

François DELERS

Danielle CHATEAU

Jean CHAMBAZ

Monique ROUSSET

Véronique CARRIÈRE

Unité mixte de recherche UMRS-872,

INSERM UPMC UPD,

Centre de Recherche des Cordeliers,

équipe 4,

15 rue de l'école de médecine,

75006 Paris,

France

$<$ veronique.carriere@crc.jussieu.fr >

Article reçu le 24 janvier 2012

accepté le 30 janvier 2012

L'intestin joue un rôle clé dans I'homéostasie lipidique via l'absorption et le transfert des lipides alimentaires à I'organisme, sous la forme de lipoprotéines riches en triglycérides (LRT). Une augmentation de la durée et du pic d'hypertriglycéridémie post-prandiale, caractérisée par une accumulation de ces LRT, contribue au développement de dyslipidémies et est un facteur de risque de maladies cardiovasculaires (Bansal et al., 2007 ; Nordestgaard et al., 2007). Si certaines des étapes responsables de la synthèse et de la sécrétion des LRT par les entérocytes (cellules absorbantes de l'intestin) sont connues, les mécanismes impliqués dans la modulation de l'absorption intestinale des lipides et son impact sur I'homéostasie lipidique restent mal caractérisés. Pourtant, la modulation de la mise à disposition des lipides par les cellules intestinales pourrait représenter de nouvelles cibles d'intervention nutritionnelle ou pharmacologique.

\begin{abstract}
Intestinal sensing of postprandial lipid micelles
Post-prandial hypertriglyceridemia is a risk factor for metabolic diseases. The intestine, through its role in alimentary lipid absorption, participates in the secretion of lipoprotein rich-triglycerides (TRL) and contributes to the increase in plasma triglyceride levels during the postprandial state. Understanding the molecular mechanisms involved in the secretion of intestinal TRL would allow the identification of new drug targets for treatment of metabolic diseases. The sensing of lipids by intestinal cells represents a promising mechanism allowing the modulation of TRL secretion. While many studies show the importance of enteroendocrine cells in the detection of alimentary lipids, several evidence suggest also the implication of enterocytes, the absorptive intestinal cells, in this process. Recent experimental results on the role of the scavenger receptor $S R-B I$ in the detection of dietary lipids, supplied in their physiological form of postprandial lipid micelles, are reviewed.
\end{abstract}

Key words: intestine, enterocytes, enteroendocrine cells, sensing, lipid micelles, SR-BI

II y a de plus en plus de données indiquant que les lipides alimentaires, en plus de leur rôle essentiel dans le maintien de l'intégrité des membranes cellulaires et le métabolisme énergétique, sont capables d'agir comme des molécules de signalisation qui vont être détectées par les cellules épithéliales intestinales et provoquer une cascade d'événements conduisant, notamment, à la diminution de la prise alimentaire et de la vidange gastrique, contrôlant ainsi I'homéostasie lipidique. Ainsi la détection des lipides alimentaires au niveau des cellules épithéliales intestinales représenterait une nouvelle étape clé de l'homéostasie lipidique.

\section{Les grandes étapes de l'absorption intestinale des lipides}

L'absorption des lipides alimentaires par les entérocytes met en jeu des processus complexes (figure 1) (pour une revue : lqbal et al., 2009). Les lipides présents dans la lumière intestinale sont présents au sein d'une structure particulière appelée micelles lipidiques. La composition de ces micelles lipidiques varie selon l'état nutritionnel. En période interprandiale, la lumière intestinale reçoit en permanence les sécrétions biliaires qui contiennent du cholestérol, des acides biliaires et des phospholipides qui se structurent pour former les micelles lipidiques interprandiales. Après un repas, les lipides alimentaires, majoritairement des triglycérides (TG), sont hydrolysés par les lipases gastriques et pancréatiques en monoglycérides et acides gras libres. Au niveau du duodénum (région proximale de l'intestin grêle), ces produits d'hydrolyse s'associent aux sécrétions biliaires (cholestérol, acides biliaires et phospholipides) pour former les micelles lipidiques postprandiales (MPP). Les lipides sont absorbés via des récepteurs spécifiques de chaque

Pour citer cet article : Beaslas O, Cueille C, Delers F, Chateau D, Chambaz J, Rousset M, Carrière V. Détection luminale des micelles lipidiques. OCL 2012 ; 19(4) : 196-199. doi : 10.1684/ocl.2012.0448 


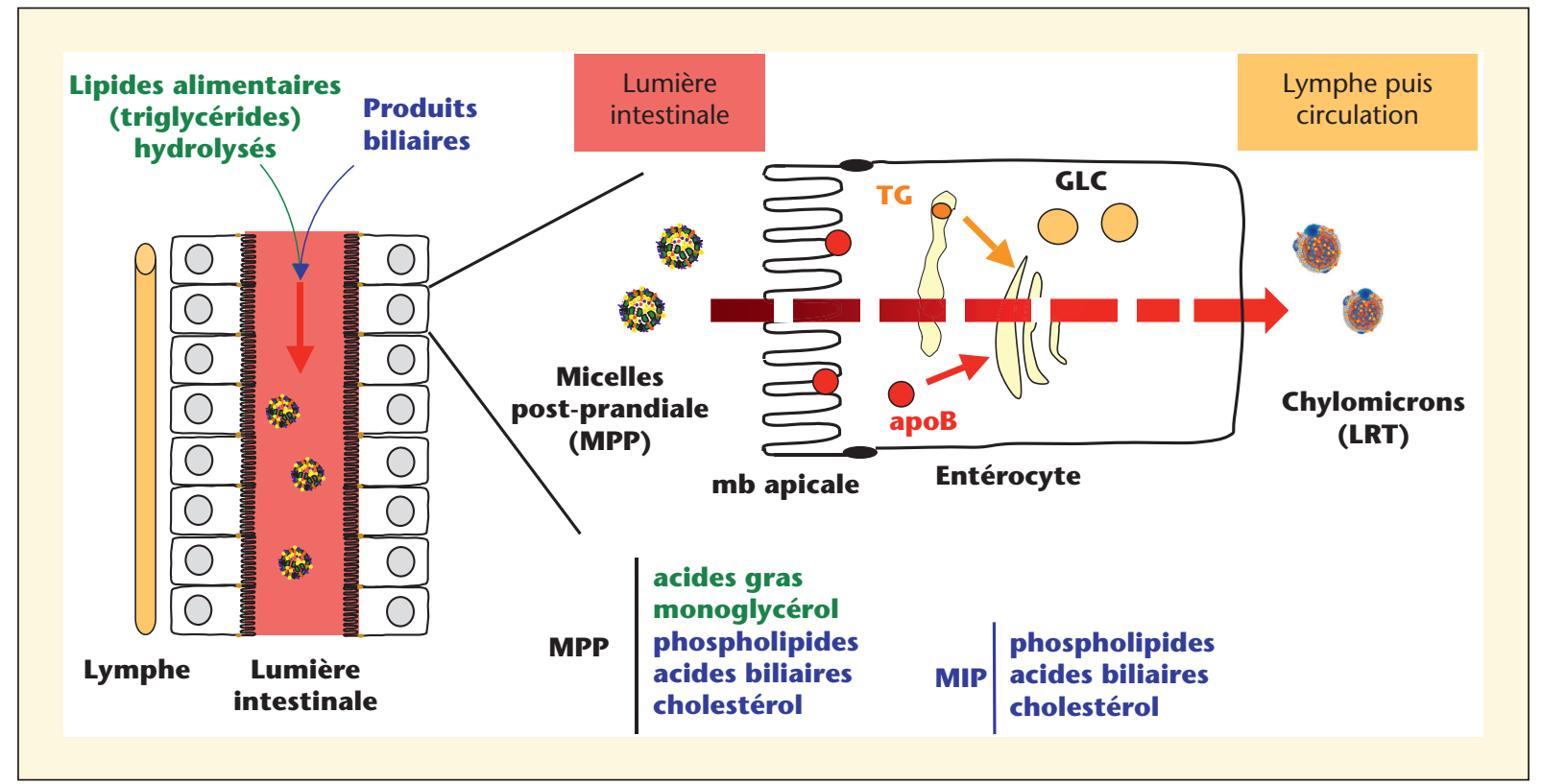

Figure 1. Les grandes étapes de l'absorption des lipides alimentaires par les entérocytes. apoB : apolipoprotéine $B, T G$ : triglycérides, LRT: lipoprotéines riches en TG, GLC: gouttelettes lipidiques cytosoliques, mb: membrane, MIP: micelles interprandiales, MPP: micelles postprandiales.

constituant ou par diffusion passive au niveau de la membrane apicale des entérocytes. Dans les entérocytes, les TG sont resynthétisés à la membrane du reticulum endoplasmique et associés aux apolipoprotéines, en particulier I'apolipoprotéine $B$ (apoB), pour former des LRT spécifiquement intestinales, les chylomicrons, qui sont sécrétés dans la lymphe puis dans la circulation générale, apportant le cholestérol et les TG nécessaires à l'organisme. En parallèle, une partie des TG est stockée transitoirement sous forme de gouttelettes lipidiques cytosoliques, avant d'être réutilisés pour la sécrétion de LRT.

La formation des micelles lipidiques post-prandiales est l'une des étapes clés de I'absorption des lipides alimentaires par l'intestin (Tso et al., 2004). Elle permet aux lipides et en particulier aux acides gras à longues chaînes, de nature très hydrophobe, de traverser la couche d'eau non agitée présente dans la lumière intestinale à proximité de la membrane apicale des entérocytes. L'action des pompes à protons de la membrane apicale des entérocytes va provoquer une acidification de cette couche d'eau non agitée entraînant la protonation des acides gras et la déstructuration des micelles lipidiques post-prandiales selon un processus appelé la dissociation micellaire. S'il est admis que les micelles lipidiques en tant qu'entités ne pénètrent pas entières dans l'entérocyte, des travaux montrent cependant que la structure des micelles lipidiques postprandiales serait détectée au niveau de la membrane apicale des entérocytes.

\section{La détection des lipides alimentaires par les cellules épithéliales intestinales : principales données actuelles}

La plupart des travaux rapportant une détection des lipides alimentaires au niveau de la muqueuse intestinale, rapportent le rôle essentiel des cellules entéroendocrines (qui représentent environ $1 \%$ de la population cellulaire de l'épithélium intestinal). II existe plusieurs types de cellules entéroendocrines qui sont spécialisés dans la sécrétion d'entéropeptides particuliers. Ainsi, les cellules entéroendocrines I sont localisées dans la partie proximale de l'intestin et sécrètent la cholécystokinine (CCK) alors que les cellules entéroendocrines $L$, présentes dans la partie distale de l'intestin, sécrètent les entéropeptides issus du clivage du proglucagon, comme le glucagon like peptide-1 et -2 (GLP-1 et GLP-2, respectivement). Les entérohormones sécrétées sont ensuite reconnues par des récepteurs spécifiques présents au niveau des terminaisons nerveuses du nerf vague. De nombreux travaux montrent que des régimes riches en lipides ou des infusions intraduodénales d'acides gras à longues chaînes sont capables de stimuler la sécrétion des entéropeptides CCK et GLP-1. Les mécanismes cellulaires et moléculaires impliqués dans la détection des acides gras et les événements de signalisation qui en découlent restent encore très mal connus (voir pour revue Little et Feinle-Bisset, 2010). Différents récepteurs à protéines $G$ comme les GPR120, 119 et 40 seraient impliqués dans la détection des acides gras au niveau des cellules entéroendocrines, mais les relais intracellulaires restent inconnus.

Différents arguments expérimentaux suggèrent un rôle des entérocytes et de la formation des chylomicrons dans la sécrétion des entéropeptides. L'entrée des acides gras au niveau des entérocytes provoquerait la formation de l'oleoylethanolamine, qui activerait le récepteur nucléaire PPARalpha, qui en retour serait 
capable d'augmenter la sécrétion d'apolipoproteine A-IV, une apolipoprotéine connue pour contrôler la prise alimentaire (pour revue Kohan et al., 2012). L'apolipoprotéine A-IV, sécrétée par les entérocytes associées aux chylomicrons en réponse à un apport lipidique, contrôlerait la sécrétion de CCK. D'autre part, les effets des acides gras sur la sécrétion des entéropeptides CCK et GLP-1 sont inhibés si on bloque I'absorption des lipides (en utilisant des inhibiteurs des lipases gastriques et pancréatiques) ou la formation des chylomicrons par les entérocytes. II est également intéressant de noter que seuls les acides gras possédant des chaînes carbonées supérieures à 12 sont capables de provoquer efficacement la sécrétion d'entérohormones. Ces acides gras à longues chaînes (>C12) sont insolubles et doivent être transportés sous forme de micelles lipidiques dans la lumière intestinale, contrairement aux acides gras à chaînes carbonées inférieures à C12 qui sont beaucoup plus hydrophiles et peuvent ainsi traverser la couche d'eau non agitée librement. De plus, les acides gras à longues chaînes seront sécrétés par les entérocytes associés aux chylomicrons contrairement aux acides gras à courtes chaînes qui sont sécrétés librement dans la circulation sanguine (pour une revue : Little et Feinle-Bisset, 2010).

L'ensemble de ces arguments suggèrent donc un rôle des entérocytes, mais aussi des micelles lipidiques, dans la détection des lipides alimentaires.

\section{Les protéines candidates de la détection des lipides alimentaires par les entérocytes}

Les protéines CD36 et SR-BI exprimées à la membrane apicale des entérocytes sont de bons candidats pour assurer le rôle de détecteurs des lipides alimentaires.

La protéine CD36 est connue pour être un transporteur d'acides gras à longues chaînes. Elle est connue pour son rôle dans la détection du "goût du gras " au niveau des papilles gustatives de la langue. Cette détection linguale des acides gras à longues chaînes provoque très rapidement une augmentation des sécrétions pancréatiques et biliaires qui ont pour but de faciliter la digestion des aliments (pour une revue : Martin et al., 2011). Un rôle de CD36 dans la détection des acides gras à longues chaînes a récemment été observé au niveau des cellules de l'épithélium intestinal. Cette détection mettrait en jeu des voies de signalisation et serait impliquée dans I'adaptation du métabolisme entérocytaire dans la production de lipoprotéines riches en triglycérides (Tran et al., 2011, voir l'article présenté dans ce numéro).

Le récepteur SR-BI (scavenger receptor class $B$ type 1) est un transporteur de cholestérol très étudié pour son rôle dans le métabolisme du cholestérol au niveau de l'organisme. SR-BI est une protéine transmembranaire, exprimée majoritairement au pôle apical des entérocytes. La découverte de la protéine NPC1L1 comme étant le transporteur responsable de l'entrée du cholestérol dans les entérocytes, a remis en cause la fonction de SR-BI dans l'épithélium intestinal qui reste encore mal connue. Des études récentes montrent cependant que SR-BI serait impliqué dans la sécrétion intestinale des lipoprotéines riches en TG.

\section{Rôle du récepteur scavenger SR-BI dans la détection des micelles lipides post-prandiales par les entérocytes}

Le rôle de SR-BI dans la détection des lipides alimentaires a été mis en évidence dans la lignée d'entérocytes humains en culture, Caco-2/C7 (Béaslas et al., 2009). Les cellules Caco-2/TC7 ont été cultivées sur filtre en présence de milieux de culture de composition différente au niveau apical et basal : au niveau basal, qui fait face au milieu intérieur in vivo, le milieu de culture est additionné de sérum; au pôle apical, qui fait face à la lumière intestinale in vivo, des micelles lipidiques post-prandiales (MPP), de composition similaire à celle retrouvée dans la lumière intestinale après un repas riche en graisse, sont ajoutées au milieu de culture. Les micelles post-prandiales sont composées des produits biliaires (cholestérol, phospholipides et acides biliaires) et des produits d'hydrolyse des triglycérides (acides gras et monoglycérol). Dans les expériences réalisées avec la lignée cellulaire Caco-2/TC7, I'acide gras utilisé est l'acide oléique. L'effet de I'apport apical des MPP a de plus été comparé à celui d'un apport de micelles lipidiques interprandiales (MIP) contenant les mêmes lipides biliaires que les MPP, mais qui sont dépourvues des produits d'hydrolyse des triglycérides.

L'apport apical de MPP provoque rapidement (dès 5 minutes) des événements de signalisation, en particulier une activation des protéines kinases ERK et p38MAPK, qui augmente avec la quantité de MPP apportée. Le deuxième événement de signalisation qui a été observé concerne le trafic intracellulaire de I'apolipoprotéine $B$, la protéine structurale des lipoprotéines riches en TG. En effet, l'apport apical de MPP est capable de provoquer la délocalisation de l'apoB présente au pôle apical des cellules vers des compartiments intracellulaires signant son engagement dans la voie sécrétoire. L'ensemble de ces effets n'est pas reproduit après un apport de MIP ou d'acide gras seul, montrant la spécificité de la signalisation induite par les MPP.

Des expériences in vitro ont montré que seules les MPP interagissent avec le récepteur SR-BI. De plus, I'apport apical de MPP provoque la concentration de SR-BI dans des régions particulières de la membrane apicale des cellules Caco-2/ TC7, ainsi que dans des domaines membranaires reconnus pour être des plateformes de signalisation, les rafts. L'implication directe de SR-BI dans la détection des micelles lipidiques postprandiales a été confirmée à l'aide d'inhibiteurs de l'activité de ce récepteur ou d'un modèle cellulaire dans lequel I'expression de SR-BI a été invalidée. En effet, I'absence de SR-BI ou son inhibition empêche la stimulation des voies de signalisation normalement induites par I'apport apical de MPP.

Un lien entre les deux événements de signalisation associés à la détection des micelles lipidiques post-prandiales a pu être mis en évidence. L'utilisation d'un inhibiteur de la voie p38MAPK bloque non seulement l'activation de la p38MAPK après un apport de MPP mais aussi le trafic intracellulaire de l'apoB.

L'ensemble des étapes de la détection des lipides alimentaires par les MPP via le récepteur SR-BI est résumé dans la figure 2 . 


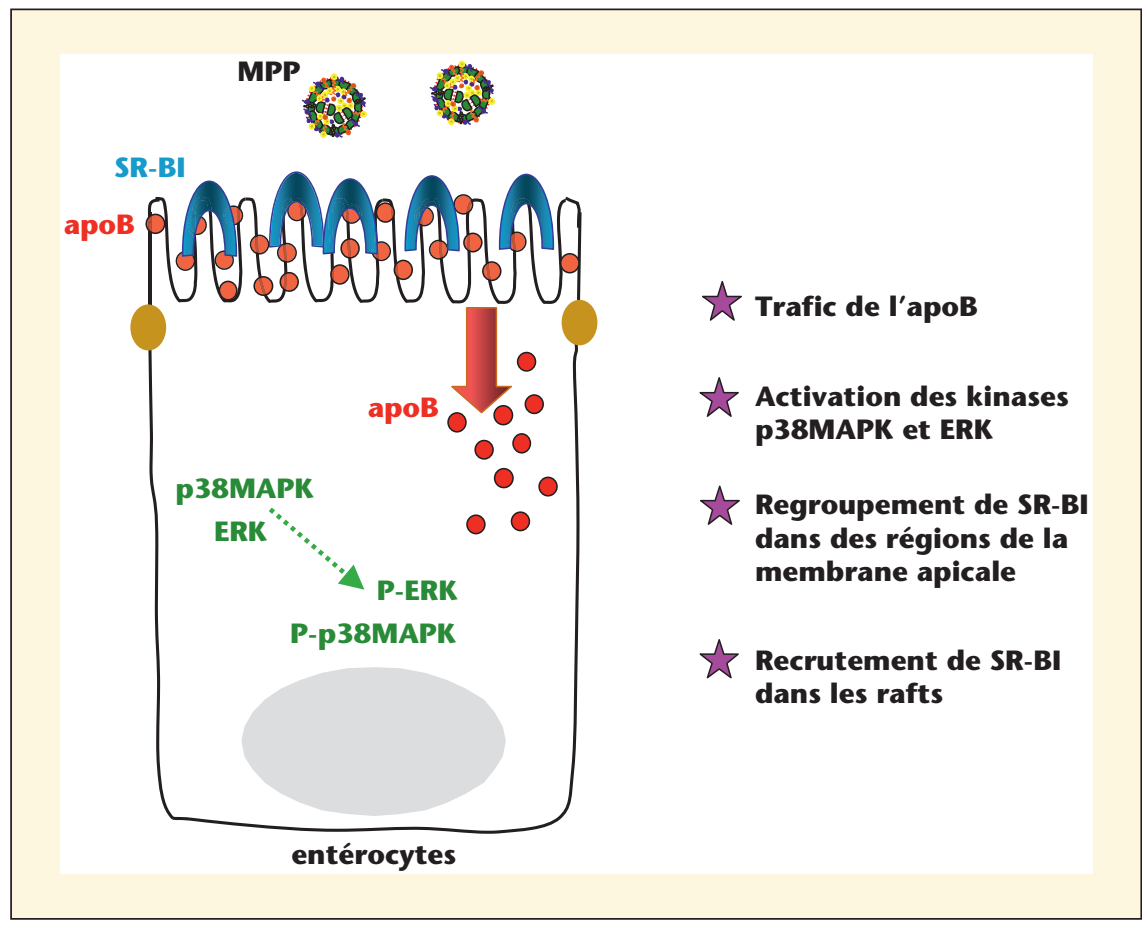

Figure 2. Evénements de signalisation associés à la détection des MPP par SR-BI dans les entérocytes. apoB : apolipoprotéine $B, M P P$ : micelles post-prandiales.

\section{Perspectives}

Les travaux sur la détection intestinale des lipides alimentaires sont actuellement en plein essor. Ils mettent en valeur l'implication d'au moins deux types cellulaires de l'épithélium intestinal, les cellules entéroendocrines et les cellules entérocytaires, ainsi que de multiples détecteurs et relais intracellulaires. De nombreuses études sont encore nécessaires pour relier entre

\section{RÉFÉRENCES}

Bansal S, Buring JE, Rifai N, Mora S, Sacks FM, Ridker PM. Fasting compared with nonfasting triglycerides and risk of cardiovascular events in women. JAMA 2007; 298 : 309-16.

Béaslas O, Cueille C, Delers F, Château D, Chambaz J, Rousset $M$, et al. Sensing of dietary lipids by enterocytes : a new role for SR-BI/Cla-1. PLoS one 2009 ; e4278.

Iqbal J, Hussain MM. Intestinal lipid absorption. Am / Physiol Endocrinol Metab 2009 ; 296 : E1183-1194.

Kohan AB, Wang F, Li X, Bradshaw S, Yang Q, Caldwell JL, et al. Apolipoprotein A-IV regulates chylomicron metabolism - mechanism and function. Am J Physiol Gastrointest Liver Physiol. 2012 (sous presse).

Little T, Feinle-Bisset C. Oral and gastrointestinal sensing of dietary fat and appetite regulation in humans: modification by diet and obesity. Frontiers in neuroscience 2010 ; $4: 178$.

Martin C, Chevrot M, Poirier H, PassilyDegrace, Niot I, Besanrd P. CD36 as a lipid sensor. Physiology \& Behavior 2011 ; $105: 36-42$.

Nordestgaard BG, Benn M, Schnohr P, Tybjaerg-Hansen A. Nonfasting triglycerides and risk of myocardial infarction, ischemic heart disease, and death in men and women. JAMA 2007 ; 298 : 299-308.

Tran TTT, Poirier $\mathrm{H}$, Clément $\mathrm{L}$, et al. Luminal lipid regulates CD36 levels and downxtream signaling to stimulate chylomicron synthesis. IBC 2011 ; 286 : 25201-10.

Tso P, Nauli A, Lo CM. Enterocyte fatty acid uptake and intestinal fatty acid-binding protein. 44th International Conference on the Bioscience of Lipids, Biochemical Society Transactions, 2004, Volume 32, part 1: 75-8. 